\title{
Threshold dynamics of a delayed predator-prey model with impulse via the basic reproduction number
}

\author{
Xiangsen Liu ${ }^{1 *}$ and Binxiang Dai ${ }^{2}$
}

\section{"Correspondence:}

xiangsen920@163.com

'Department of Mathematics,

North University of China, Taiyuan,

P.R. China

Full list of author information is

available at the end of the article

\section{Springer}

\begin{abstract}
In this paper, we study a delayed predator-prey model with impulse and, in particular, the existence of the predator-free periodic solution. We employ the approach and techniques coming from epidemiology and calculate the basic reproduction number for the predator. Using the basic reproduction number, we consider the global attraction of the predator-free periodic solution and uniform persistence of the predator. Our results improve the results by Li and Liu (Adv. Differ. Equ. 2016:42, 2016), where they left the open problem of finding a threshold value that determines the eradication and uniform persistence of the predator. Furthermore, we give some numerical simulations to illustrate our results.
\end{abstract}

Keywords: Predator-prey model; Delay; Impulse; Basic reproduction number

\section{Introduction}

In the natural world, many species usually pass through a number of stages during their life cycle. So it is practical to introduce time delay into models of theoretical ecology. In particular, it is often important to take into account the processes of gestation and maturation to make an abstract model more biologically realistic [2-4]. The characteristic property of population models with time delay is their oscillatory behavior: for a sufficiently large maturation period, an initially stable equilibrium becomes unstable, and the system exhibits sustained oscillations [2, 5]. Additionally, impulsive differential equations have been extensively used as models in biology, physics, chemistry, engineering, and other sciences with particular emphasis on population dynamics [6-9]. In [9] the authors discussed an impulsive predator-prey system with stage structure and generalized functional response. Sufficient conditions are established for the existence of a predator-free positive periodic solution and the permanence of the system. Numerical simulation shows that impulses and functional response affect the dynamics of the system.

Hence time delay and impulse are incorporated into predator-prey models, which greatly enrich biologic background. However, the systems become so complicated that they cause great difficulties to study the models. The investigation of impulsive delaydifferential equations is inchoate and focuses on theoretical analysis [10-12]. In [1] the

(c) The Author(s) 2018. This article is distributed under the terms of the Creative Commons Attribution 4.0 International License (http://creativecommons.org/licenses/by/4.0/), which permits unrestricted use, distribution, and reproduction in any medium, provided you give appropriate credit to the original author(s) and the source, provide a link to the Creative Commons license, and indicate if changes were made. 
authors considered the following delayed model with impulse:

$$
\left\{\begin{array}{l}
x^{\prime}(t)=r x(t)\left(1-\frac{x(t)}{K}\right)-\frac{\alpha x^{2}(t) y(t)}{1+\beta x^{2}(t)}, \\
y^{\prime}(t)=\frac{k \alpha x^{2}(t-\tau) y(t-\tau)}{1+\beta x^{2}(t-\tau)}-d y(t), \\
\Delta x(t)=-p x(t), \\
\Delta y(t)=0,
\end{array}\right\} \quad t=n T,
$$

where $x(t)$ and $y(t)$ denote the population densities of prey and predator at time $t$, respectively, $r, K, \alpha, \beta, k, d$ are positive, $r$ is the intrinsic rate of increase of the prey, $K$ is the carrying capacity of the prey, $\alpha$ is the predation coefficient of the predator, which reflects the size of the predator's ability, $\beta$ is the predation regulation factor (saturation factor) of the predator, $d$ is the death rate of the predator, $k(0<k<1)$ is the rate of conversing prey into predator, $\tau>0$ denotes a time delay due to the gestation of the predator, $\Delta x(t)=x\left(t^{+}\right)-x(t), \Delta y(t)=y\left(t^{+}\right)-y(t), T$ is the impulsive period, $n \in \mathbb{N}_{+}=\{1,2, \ldots\}$, and $p>0$ is the proportionality constant, which represents the rate of mortality due to the applied pesticide. The initial conditions for system (1.1) are

$$
\left(\phi_{1}(s), \phi_{2}(s)\right) \in C\left([-\tau, 0], \mathbb{R}_{+}^{2}\right), \quad \phi_{i}(0)>0(i=1,2) .
$$

In [1], sufficient conditions for the global attraction of a predator-free periodic solution are obtained by the theory of impulsive differential equations, that is, $T<T_{1}^{*}$. The conditions for the permanence of the system are investigated, that is, $T>T_{2}^{*}$. Note that $T_{1}^{*}<T_{2}^{*}$ always holds. It is obvious that if $T \in\left(T_{1}^{*}, T_{2}^{*}\right)$, then we cannot determine whether the predator can persist or not. In the present paper, we give a thorough global dynamics of (1.1), which completely solves the question left in [1]. To do this, we employ the approach coming from epidemiology [13]. As far as we know, there are no papers employing this approach in ecology. Throughout the present paper, roughly speaking, the basic reproduction number $R_{0}$ may be thought as the number of predators one predator gives rise during its life, when introduced in a prey population [14]. A similar threshold value for the coexistence of a predator-prey system has previously been formulated and explained by Pielou [15], among others but, to the best of our knowledge, has not been termed a "basic reproduction number." In ecology, many authors have investigated the autonomous predator-prey systems using the basic reproduction number [16, 17]. For example, in [16] the authors considered a stage-structured predator-prey model with nonlinear predation rate. They discussed the stability of the system using the basic reproduction number of the predator population. In contrast, there have been few papers discussing the nonautonomous, delayed, or impulsive predator-prey systems using the basic reproduction number (except for [18]). In [18] the authors considered an ecoepidemiological model with Holling type-III functional response and time delay. They used the ecological and disease basic reproduction numbers to determine the persistence of the system. In this paper, using the basic reproduction number of the predator population and approach in [13], we wish to find a threshold value to determine whether the predator can exist or not.

The remainder of this paper is organized as follows. In the next section, we discuss the existence of a predator-free periodic solution and boundedness of system (1.1). In Sect. 3, 
we employ the approach coming from epidemiology and calculate the basic reproduction number for the predator. In Sect. 4, using the basic reproduction number, we consider the global attraction of the predator-free periodic solution and persistence of the predator in (1.1). In Sect. 5, we give some numerical simulations to illustrate our results. Finally, we give some concluding remarks.

\section{The existence of a predator-free periodic solution and boundedness of system (1.1)}

In this section, we investigate the existence of a predator-free periodic solution of system (1.1). In this case, the predator population is entirely absent from the population permanently, that is, $y(t)=0, t \geq 0$. System (1.1) yields

$$
\begin{cases}x^{\prime}(t)=r x(t)\left(1-\frac{x(t)}{K}\right), & t \neq n T, \\ \Delta x(t)=-p x(t), & t=n T .\end{cases}
$$

For system (2.1), we have the following result.

Lemma 2.1 ([1]) If $(1-p) e^{r T}>1$, then system (2.1) has the unique positive periodic solution

$$
x^{*}(t)=\frac{x_{0}^{*}}{\left(1-x_{0}^{*} / K\right) e^{-r(t-n T)}+x_{0}^{*} / K}, \quad t \in(n T,(n+1) T],
$$

which is globally asymptotically stable, where

$$
x_{0}^{*}=\frac{K\left((1-p) e^{r T}-1\right)}{e^{r T}-1} .
$$

According to Lemma 2.1, we obtain the following result.

Theorem 2.1 If $(1-p) e^{r T}>1$, then system (1.1) has a predator-free periodic solution $\left(x^{*}(t), 0\right)$, where $x^{*}(t)$ is shown in (2.2).

Next, we will show that all solutions of (1.1) are uniformly upper bounded.

Theorem 2.2 If $(1-p) e^{r T}>1$, then all solutions of (1.1) are uniformly upper bounded.

Proof From the first and third equations of (1.1) we have

$$
\begin{cases}x^{\prime}(t) \leq r x(t)\left(1-\frac{x(t)}{K}\right), & t \neq n T, \\ \Delta x(t)=-p x(t), & t=n T .\end{cases}
$$

Consider the following impulsive comparison system:

$$
\begin{cases}z_{1}^{\prime}(t)=r z_{1}(t)\left(1-\frac{z_{1}(t)}{K}\right), & t \neq n T, \\ \Delta z_{1}(t)=-p z_{1}(t), & t=n T .\end{cases}
$$

By Lemma 2.1 we obtain that system (2.4) has a globally asymptotically stable periodic solution $z_{1}^{*}(t)=x^{*}(t)$, where $x^{*}(t)$ is defined in (2.2). In view of the comparison principle, 
for given $\epsilon_{0}>0$, there exists $T_{1}>0$ such that, for $t>T_{1}$,

$$
x(t) \leq z_{1}(t) \leq x^{*}(t)+\epsilon_{0} \leq \frac{x_{0}^{*}}{\left(1-x_{0}^{*} / K\right) e^{-r T}+x_{0}^{*} / K}+\epsilon_{0} \triangleq M_{1} .
$$

Let $V(t)=k x(t-\tau)+y(t)$. Then, for $t>T_{1}+\tau$, we have

$$
\begin{aligned}
V^{\prime}(t) & =k r x(t-\tau)\left(1-\frac{x(t-\tau)}{K}\right)-d y(t) \\
& \leq k r x(t-\tau)-d y(t) \\
& =(k r+k d) x(t-\tau)-d(k x(t-\tau)+y(t)) \\
& \leq k(r+d) M_{1}-d V(t)
\end{aligned}
$$

and

$$
\begin{aligned}
V\left((n T+\tau)^{+}\right) & =k x\left(n T^{+}\right)+y\left((n T+\tau)^{+}\right) \\
& =k(1-p) x(n T)+y(n T+\tau) \\
& \leq k x(n T)+y(n T+\tau) \\
& =V(n T+\tau) .
\end{aligned}
$$

Consider the following impulsive comparison system:

$$
\begin{cases}V_{1}^{\prime}(t)=k(r+d) M_{1}-d V_{1}(t), & t \neq n T+\tau \\ \Delta V_{1}(t)=0, & t=n T+\tau\end{cases}
$$

It is clear that

$$
\lim _{t \rightarrow+\infty} V_{1}(t)=\frac{k(r+d) M_{1}}{d}
$$

By the comparison principle, for $\epsilon_{0}>0$, there exists $T_{2}>T_{1}+\tau$ such that, for $t>T_{2}$, we get

$$
V(t) \leq V_{1}(t) \leq \frac{k(r+d) M_{1}}{d}+\epsilon_{0} \triangleq M_{2}
$$

The proof is completed.

Thus the dynamics of system (1.1) can be analyzed in the following bounded feasible region:

$$
\Gamma=\left\{(x(t), y(t)) \mid 0 \leq x(t) \leq M_{1}, 0 \leq y(t) \leq M_{2}\right\}
$$

where $M_{1}$ and $M_{2}$ are defined in (2.5) and (2.6), respectively. 


\section{The basic reproduction number of the predator}

In this section, we introduce the basic reproduction number $R_{0}$ of the predator for system (1.1) using the next generation operators approach in [19]. We linearize system (1.1) at $\left(x^{*}(t), 0\right)$ and get

$$
\begin{aligned}
y^{\prime}(t) & =\frac{k \alpha x^{* 2}(t-\tau)}{1+\beta x^{* 2}(t-\tau)} y(t-\tau)-d y(t) \\
& =g(t) y(t-\tau)-d y(t),
\end{aligned}
$$

where

$$
g(t)=\frac{k \alpha x^{* 2}(t-\tau)}{1+\beta x^{* 2}(t-\tau)}
$$

From (3.1) we obtain

$$
y(t)=y(0) e^{-d t}+e^{-d t} \int_{0}^{t} g(s) y(s-\tau) e^{d s} \mathrm{~d} s .
$$

Let $y_{1}(t)=g(t) y(t-\tau)$. Then

$$
y_{1}(t)=y(0) g(t) e^{-d(t-\tau)}+e^{-d(t-\tau)} g(t) \int_{0}^{t-\tau} y_{1}(s) e^{d s} \mathrm{~d} s .
$$

Making the change of variable $s=t-\delta$, we get

$$
\begin{aligned}
y_{1}(t) & =y(0) g(t) e^{-d(t-\tau)}+g(t) e^{-d(t-\tau)} \int_{\tau}^{t} y_{1}(t-\delta) e^{d(t-\delta)} \mathrm{d} \delta \\
& =y(0) g(t) e^{-d(t-\tau)}+g(t) \int_{\tau}^{t} y_{1}(t-\delta) e^{-d(\delta-\tau)} \mathrm{d} \delta \\
& =y_{10}(t)+\int_{\tau}^{t} \bar{K}(t, \delta) y_{1}(t-\delta) \mathrm{d} \delta,
\end{aligned}
$$

where

$$
y_{10}(t)=y(0) g(t) e^{-d(t-\tau)}
$$

and

$$
\bar{K}(t, \delta)= \begin{cases}0, & \delta<\tau, \\ g(t) e^{-d(\delta-\tau)}, & \delta \geq \tau .\end{cases}
$$

Let $C_{T}$ be the ordered Banach space of all continuous $T$-periodic functions from $\mathbb{R}$ to $\mathbb{R}$ equipped with the maximum norm $\|\cdot\|$ and the positive cone $C_{T}^{+}=\left\{\psi \in C_{T} \mid \psi(t) \geq\right.$ $0, t \in \mathbb{R}\}$. Then we can define the linear operator $L: C_{T} \rightarrow C_{T}$ by

$$
L: v(t) \mapsto \int_{0}^{+\infty} \bar{K}(t, \delta) v(t-\delta) \mathrm{d} \delta .
$$


Following [19], the basic reproduction number of the predator is defined as $R_{0} \triangleq r(L)$, the spectral radius of $L$.

We see that the basic reproduction number $R_{0}=r(L)$ can be obtained by solving the eigenvalue problem

$$
g(t) \int_{\tau}^{+\infty} u(t-\delta) e^{-d(\delta-\tau)} \mathrm{d} \delta=R_{0} u(t), \quad u \in C_{T} .
$$

Differentiating Eq. (3.3) and then integrating by parts, we have

$$
\begin{aligned}
R_{0} u^{\prime}(t) & =g^{\prime}(t) \int_{\tau}^{+\infty} u(t-\delta) e^{-d(\delta-\tau)} \mathrm{d} \delta+g(t) \int_{\tau}^{+\infty} u^{\prime}(t-\delta) e^{-d(\delta-\tau)} \mathrm{d} \delta \\
& =g^{\prime}(t) \frac{R_{0} u(t)}{g(t)}-g(t) \int_{\tau}^{+\infty} e^{-d(\delta-\tau)} \mathrm{d} u(t-\delta) \\
& =g^{\prime}(t) \frac{R_{0} u(t)}{g(t)}+g(t) u(t-\tau)-d R_{0} u(t) .
\end{aligned}
$$

This equation can be rewritten as

$$
\frac{u^{\prime}(t)}{u(t)}=\frac{g^{\prime}(t)}{g(t)}+\frac{g(t) u(t-\tau)}{R_{0} u(t)}-d
$$

Note that $u(0)=u(T)$ and $g(0)=g(T)$. Integrating both sides of (3.4) from 0 to $T$ yields

$$
R_{0}=\frac{\int_{0}^{T}[g(t) u(t-\tau) / u(t)] \mathrm{d} t}{d T} .
$$

Obviously, there is no explicit formula for $R_{0}$ when $\tau \neq 0$.

Remark 3.1 When $\tau=0$, system (1.1) reduces to a periodic system of ordinary differential equations. The corresponding basic reproduction number $R_{0}$ becomes

$$
R_{0}=\frac{\int_{0}^{T} g(t) \mathrm{d} t}{d T} .
$$

\section{The global dynamics of system (1.1)}

In this section, we study the global dynamics of system (1.1) in terms of its basic reproduction number $R_{0}$. To this end, we first introduce some lemmas for our main results.

For any $\psi \in C([-\tau, 0], \mathbb{R})$, let $P(t) \psi=u_{t}(\psi)$ be the unique solution of (3.1) satisfying $u_{0}=\psi$. Then $P \triangleq P(T)$ is the Poincaré map of (3.1).

Lemma 4.1 ([20]) Let $r(P)$ be the spectral radius of $P$. Then the following statements are valid:

(1) $R_{0}=1$ if and only if $r(P)=1$;

(2) $R_{0}>1$ if and only if $r(P)>1$;

(3) $R_{0}<1$ if and only if $r(P)<1$.

Consider the following linear equation with time delay:

$$
u^{\prime}(t)=a(t) u(t)+b(t) u(t-\tau)
$$


where $a(t) \in C_{T}$, and $b(t)$ is a piecewise left-continuous $T$-periodic function with discontinuous points of first kind at $n T$ such that $b(t)>0, t \geq 0$. By applying the method of steps it is easy to verify that, for any $\phi \in C_{+}=C\left([-\tau, 0], \mathbb{R}_{+}\right)$, system (4.1) has a unique continuous solution $u(t, \phi)$ on $[-\tau,+\infty)$ with $u_{0}=\phi$. Let $P$ be the Poincaré map associated with (4.1) on $C_{+}$, that is, $P(\phi)=u_{T}(\phi)$. Then we have the following result.

Lemma 4.2 [13]. Let $\mu=\ln \frac{r(P)}{T}$. Then there exists a positive T-periodic function $v(t)$ such that $e^{\mu t} v(t)$ is a solution of (4.1).

For the predator-free periodic solution $\left(x^{*}(t), 0\right)$ of $(1.1)$, we have the following result.

Theorem 4.1 Assume that $(1-p) e^{r T}>1$. If $R_{0}<1$, then the predator-free periodic solution $\left(x^{*}(t), 0\right)$ of $(1.1)$ is globally attracting, where $R_{0}$ is defined in (3.5).

Proof Let $P_{\epsilon}$ be the Poincaré map of the following perturbed linear equation:

$$
y_{2}^{\prime}(t)=\frac{k \alpha\left(x^{*}(t-\tau)+\epsilon\right)^{2}}{1+\beta\left(x^{*}(t-\tau)+\epsilon\right)^{2}} y_{2}(t-\tau)-d y_{2}(t) .
$$

By Lemma 4.1 we see that $R_{0}<1$ if and only if $r(P)<1$, where $P$ is the Poincaré map of (3.1). Since $\lim _{\epsilon \rightarrow 0} r\left(P_{\epsilon}\right)=r(P)<1$, we may fix a small enough $\epsilon>0$ such that $r\left(P_{\epsilon}\right)<1$. By Lemma 4.2 there is a positive $T$-periodic function $v_{\epsilon}(t)$ such that $e^{\mu_{\epsilon} t} v_{\epsilon}(t)$ is a positive solution of (4.2), where $\mu_{\epsilon}=\frac{\ln r\left(P_{\epsilon}\right)}{T}<0$.

According to the proof of Theorem 2.2, for given $\epsilon>0$, there exists $T_{3}>0$ such that, for $t>T_{3}$,

$$
x(t) \leq x^{*}(t)+\epsilon,
$$

where $x^{*}(t)$ is defined in (2.2).

By the second equation of (1.1), for $t>T_{3}+\tau$, we get

$$
y^{\prime}(t) \leq \frac{k \alpha\left(x^{*}(t-\tau)+\epsilon\right)^{2}}{1+\beta\left(x^{*}(t-\tau)+\epsilon\right)^{2}} y(t-\tau)-d y(t) .
$$

Choose a sufficiently large $A>0$ such that $y(t) \leq A e^{\mu_{\epsilon} t} v_{\epsilon}(t), t \in\left[T_{3}, T_{3}+\tau\right]$, where $A e^{\mu_{\epsilon} t} v_{\epsilon}(t)$ is a positive solution of (4.2). By the comparison principle we have

$$
y(t) \leq A e^{\mu_{\epsilon} t} v_{\epsilon}(t), \quad t \geq T_{3}+\tau .
$$

Since $\mu_{\epsilon}<0, \lim _{t \rightarrow+\infty} y(t)=0$.

Then for a sufficiently small $\epsilon_{1}>0\left(\epsilon_{1}<\frac{r}{\alpha M_{1}}\right.$, where $M_{1}$ is shown in (2.5)), there exists $T_{4}>0$ such that, for $t>T_{4}$, we obtain

$$
0<y(t)<\epsilon_{1}
$$

From the first and third equations of (1.1), for $t>\max \left\{T_{1}, T_{4}\right\}$, we have

$$
\begin{cases}x^{\prime}(t) \geq x(t)\left(r-\alpha M_{1} \epsilon_{1}-\frac{r}{K} x(t)\right), & t \neq n T, \\ \Delta x(t)=-p x(t), & t=n T .\end{cases}
$$


Consider the following impulsive comparison system:

$$
\begin{cases}z_{2}^{\prime}(t)=z_{2}(t)\left(r-\alpha M_{1} \epsilon_{1}-\frac{r}{K} z_{2}(t)\right), & t \neq n T, \\ \Delta z_{2}(t)=-p z_{2}(t), & t=n T .\end{cases}
$$

Since $\epsilon_{1}<\frac{r}{\alpha M_{1}}$, it is obvious that $r-\alpha M_{1} \epsilon_{1}>0$. According to Lemma 2.1, we find that system (4.4) has a globally asymptotically stable periodic solution

$$
z_{2}^{*}(t)=\frac{z_{2}^{*}(0+)}{\left(1-r z_{2}^{*}(0+) /\left(K r_{1}\right)\right) e^{-r_{1}(t-n T)}+r z_{2}^{*}(0+) /\left(K r_{1}\right)}, \quad n T<t \leq(n+1) T,
$$

with

$$
z_{2}^{*}(0+)=\frac{K r_{1}\left((1-p) e^{r_{1} T}-1\right)}{r\left(e^{r_{1} T}-1\right)}, \quad r_{1}=r-\alpha M_{1} \epsilon_{1}
$$

By the comparison principle, for $x(0+) \geq z_{2}(0+)$ and $t>\max \left\{T_{1}, T_{4}\right\}$, we have $x(t) \geq z_{2}(t)$, and $z_{2}(t)-z_{2}^{*}(t) \rightarrow 0$ as $t \rightarrow+\infty$. Meanwhile, $z_{2}^{*}(t)-x^{*}(t) \rightarrow 0$ as $\epsilon_{1} \rightarrow 0$. Based on this analysis and (4.3), we see that $x(t)-x^{*}(t) \rightarrow 0$ as $t \rightarrow+\infty$. Therefore the predator-free periodic solution $\left(x^{*}(t), 0\right)$ of $(1.1)$ is globally attracting. The proof is completed.

Theorem 4.2 Let $(1-p) e^{r T}>1$. If $R_{0}>1$, then there exists $q>0$ such that every positive solution $(x(t), y(t))$ of $(1.1)$ satisfies $y(t) \geq q$ for t large enough.

Proof Let $M_{\eta}$ be the Poincaré map of the perturbed equation

$$
\bar{y}^{\prime}(t)=\frac{k \alpha\left(x^{*}(t-\tau)-\eta\right)^{2}}{1+\beta\left(x^{*}(t-\tau)-\eta\right)^{2}} \bar{y}(t-\tau)-d \bar{y}(t)
$$

Since $\lim _{\eta \rightarrow 0} r\left(M_{\eta}\right)=r(P)>1$, we can fix a small positive number $\eta$ such that $r\left(M_{\eta}\right)>1$ and $\eta<\inf _{t \geq 0} x^{*}(t)$.

By Lemma 4.2 there is a positive $T$-periodic function $v_{\eta}(t)$ such that $e^{\mu_{\eta} t} v_{\eta}(t)$ is a positive solution of (4.5), where $\mu_{\eta}=\frac{\ln r\left(M_{\eta}\right)}{T}>0$.

Consider the impulsive comparison system

$$
\begin{cases}\bar{x}^{\prime}(t)=\bar{x}(t)\left(r-\alpha M_{1} \bar{\epsilon}-\frac{r}{K} \bar{x}(t)\right), & t \neq n T, \\ \Delta \bar{x}(t)=-p \bar{x}(t), & t=n T .\end{cases}
$$

where $0<\bar{\epsilon}<\frac{r}{\alpha M_{1}}$, and $M_{1}$ is shown in (2.5).

Using Lemma 2.1, we see that system (4.6) admits a positive periodic solution $x_{\bar{\epsilon}}^{*}(t)$, which is globally asymptotically stable, and $\lim _{\bar{\epsilon} \rightarrow 0} x_{\bar{\epsilon}}^{*}(t)=x^{*}(t)$. Hence we can fix a small number $\bar{\epsilon} \in\left(0, \frac{r}{\alpha M_{1}}\right)$ such that, for $t \geq 0$,

$$
x_{\bar{\epsilon}}^{*}(t)>x^{*}(t)-\frac{\eta}{2} .
$$

Fix a positive number $\gamma$ such that $\gamma<\min \{\eta, \bar{\epsilon}\}$. We now claim that, for any $t_{0}>0$, it is impossible that $y(t)<\gamma$ for all $t>t_{0}$. Suppose by contradiction that there is $t_{0}>0$ such that 
$y(t)<\gamma$ for $t>t_{0}$. It follows from the first equation of (1.1) that, for $t>t_{0}$,

$$
\begin{aligned}
x^{\prime}(t) & \geq r x(t)\left(1-\frac{x(t)}{K}\right)-\alpha M_{1} \gamma x(t) \\
& \geq x(t)\left(r-\alpha M_{1} \bar{\epsilon}-\frac{r x(t)}{K}\right) .
\end{aligned}
$$

Therefore we have

$$
\begin{cases}x^{\prime}(t) \geq x(t)\left(r-\alpha M_{1} \bar{\epsilon}-\frac{r x(t)}{K}\right), & t \neq n T, \\ \Delta x(t)=-p x(t), & t=n T .\end{cases}
$$

By system (4.6) and the comparison principle there exists $t_{1}>t_{0}$ such that, for $t>t_{1}$ and $x(0+) \geq \bar{x}(0+)$,

$$
x(t) \geq \bar{x}(t)>x_{\bar{\epsilon}}^{*}(t)-\frac{\eta}{2} .
$$

Using (4.7) and (4.8), for $t>t_{1}$, we get

$$
x(t)>x^{*}(t)-\eta
$$

From (4.9) and the second equation of (1.1), for $t>t_{2} \triangleq t_{1}+\tau$, we have

$$
y^{\prime}(t) \geq \frac{k \alpha\left(x^{*}(t-\tau)-\eta\right)^{2}}{1+\beta\left(x^{*}(t-\tau)-\eta\right)^{2}} y(t-\tau)-d y(t) .
$$

Choose a number $m_{1}>0$ such that

$$
y(t) \geq m_{1} e^{\mu_{\eta} t} v_{\eta}(t), \quad t \in\left[t_{1}, t_{2}\right],
$$

and

$$
m_{1} e^{\mu_{\eta} t} v_{\eta}(t)<\gamma, \quad t \in\left[t_{1}, t_{2}\right]
$$

where $e^{\mu_{\eta} t} v_{\eta}(t)$ is a positive solution of (4.5) and $\mu_{\eta}>0$. Using (4.10), (4.11), (4.12), and the comparison theorem, there must be $t_{3}>t_{2}$ such that $\gamma<y\left(t_{3}\right)<\bar{\epsilon}$, which is a contradiction.

From the above claim we discuss the following two possibilities:

(H1) $y(t) \geq \gamma$ for all large $t$.

(H2) $y(t)$ oscillates about $\gamma$ for all large $t$.

Let $q=\min \left\{\frac{\gamma}{2}, q_{2}\right\}$, where

$$
q_{2}=\frac{q_{1} \min _{t \in[0, T]} v_{\eta}(t)}{e^{\mu_{\eta} \tau} \max _{t \in[0, T]} v_{\eta}(t)}
$$

with $q_{1}=\gamma e^{-d \tau}$.

We hope to show that $y(t) \geq q$ for $t$ large enough. Obviously, we only need to consider case (H2). Let $\underline{t}$ and $\bar{t}$ satisfy

$$
y(\underline{t})=y(\bar{t})=\gamma, \quad y(t)<\gamma, \quad t \in[\underline{t}, \bar{t}],
$$


where $\underline{t}$ is sufficiently large such that, for $t \in[\underline{t}, \bar{t}]$,

$$
x(t)>x^{*}(t)-\eta \text {. }
$$

Note that the function $y(t)$ for $t \geq 0$ is uniformly continuous since its derivative is bounded for all $t \geq 0$. Hence there exists $T^{\prime}\left(0<T^{\prime}<\tau\right.$ is independent of the choice of t) such that $y(t)>\frac{\gamma}{2}$ for $t \in\left[\underline{t}, \underline{t}+T^{\prime}\right]$. Let us consider the following three cases:

Case $\left(B_{1}\right) \bar{t}-\underline{t} \leq T^{\prime}$. Then $y(t)>\frac{\gamma}{2}$ for all $t \in[\underline{t}, \bar{t}]$.

Case $\left(B_{2}\right) T^{\prime}<\bar{t}-\underline{t} \leq \tau$.

By the second equation of (1.1) we have

$$
y^{\prime}(t) \geq-d y(t)
$$

Using the comparison principle, for $t \in[\underline{t}, \bar{t}]$, we get

$$
\begin{aligned}
y(t) & \geq y(\underline{t}) e^{-d(t-\underline{t})} \\
& \geq \gamma e^{-d \tau} \triangleq q_{1} .
\end{aligned}
$$

Therefore, in this case, $y(t) \geq q_{1}$ for $t \in[t, \bar{t}]$.

Case $\left(B_{3}\right) \bar{t}-\underline{t}>\tau$

Similarly to the discussion of case $\left(B_{2}\right)$, we obtain $y(t) \geq q_{1}$ for $t \in[\underline{t}, \underline{t}+\tau]$. If $y(t) \geq q_{1}$ for $t \in[\underline{t}+\tau, \bar{t}]$, then our aim is reached. Suppose not. Then there exists $\bar{T}_{1}>0$ such that

$$
\begin{aligned}
& y(t) \geq q_{1}, \quad t \in\left[\underline{t}, \underline{t}+\tau+\bar{T}_{1}\right], \\
& y\left(\underline{t}+\tau+\bar{T}_{1}\right)=q_{1}, \quad y(t)<q_{1}, \quad 0<t-\left(\underline{t}+\tau+\bar{T}_{1}\right) \ll 1 .
\end{aligned}
$$

By (4.14) and the second equation of system (1.1), for $t \in[t, \bar{t}]$, we have

$$
y^{\prime}(t) \geq \frac{k \alpha\left(x^{*}(t-\tau)-\eta\right)^{2}}{1+\beta\left(x^{*}(t-\tau)-\eta\right)^{2}} y(t-\tau)-d y(t) .
$$

Choose a number $m_{2}=q_{1}\left[e^{\mu_{\eta}\left(t+\tau+\bar{T}_{1}\right)} \max _{t \in[0, T]} v_{\eta}(t)\right]^{-1}>0$. It is clear that, for $t \in[\underline{t}+$ $\left.\bar{T}_{1}, \underline{t}+\tau+\bar{T}_{1}\right]$,

$$
m_{2} e^{\mu_{\eta} t} v_{\eta}(t) \leq q_{1} \leq y(t)
$$

and for $t \in\left[\underline{t}+\bar{T}_{1}, \bar{t}\right]$,

$$
m_{2} e^{\mu_{\eta} t} v_{\eta}(t) \geq m_{2} e^{\mu_{\eta}\left(t+\bar{T}_{1}\right)} \min _{t \in[0, T]} v_{\eta}(t)=\frac{q_{1} \min _{t \in[0, T]} v_{\eta}(t)}{e^{\mu_{\eta} \tau} \max _{t \in[0, T]} v_{\eta}(t)}=q_{2}>0 .
$$

The comparison theorem implies that, for $t \in\left[\underline{t}+\bar{T}_{1}, \bar{t}\right]$,

$$
y(t) \geq q_{2}
$$

Thus $y(t) \geq q_{2}$ for $t \in[\underline{t}, \bar{t}]$.

Consequently, we get $y(t) \geq q$ for $t \in[\underline{t}, \bar{t}]$. Since this kind of interval $[\underline{t}, \bar{t}]$ is chosen arbitrarily, we get $y(t) \geq q$ for $t$ large enough. This completes the proof. 


\section{Numerical simulation}

In this section, we give phase portraits of system (1.1) to illustrate the above theoretical analysis using numerical simulations. We consider the following delayed model with impulse:

$$
\left\{\begin{array}{l}
x^{\prime}(t)=1.5 x(t)\left(1-\frac{x(t)}{5}\right)-\frac{0.75 x^{2}(t) y(t)}{1+x^{2}(t)}, \\
y^{\prime}(t)=\frac{3 x^{2}(t-\tau) y(t-\tau)}{8\left(1+x^{2}(t-\tau)\right)}-0.24 y(t), \\
\left.\begin{array}{l}
\Delta x(t)=-0.5 x(t), \\
\Delta y(t)=0,
\end{array}\right\} \quad t=n T,
\end{array}\right\} \quad t \neq n T,
$$

where $\tau=0.5$ and the initial conditions $\left(\phi_{1}(t), \phi_{2}(t)\right)=(1,1), t \in[-\tau, 0]$. In [1] the authors got the thresholds $T_{1}^{*} \approx 0.5734$ and $T_{2}^{*} \approx 0.7634$ for system (5.1). Meanwhile, they concluded that if $T<T_{1}^{*}$, then the predator-free periodic solution is globally attractive, and if $T>T_{2}^{*}$, then the predator will persist. However, if $T \in\left(T_{1}^{*}, T_{2}^{*}\right)$, then they could not determine whether the predator can persist or not. In the present paper, we completely solve this question.

By Eq. (3.3), for system (5.1), the following eigenvalue problem can be obtained:

$$
g_{1}(t) \int_{0.5}^{+\infty} u(t-\delta) e^{-0.24(\delta-0.5)} \mathrm{d} \delta=R_{0} u(t), \quad u \in C_{T},
$$

where $R_{0}$ and $u(t)$ are the eigenvalue and eigenvector of Eq. (5.2), respectively, and

$$
g_{1}(t)=\frac{3\left(5 e^{1.5 T}-10\right)^{2}}{8\left[\left(e^{-1.5(t-0.5-(n+1) T)}+e^{1.5 T}-2\right)^{2}+\left(5 e^{1.5 T}-10\right)^{2}\right]} .
$$

So we have

$$
\begin{aligned}
R_{0}= & \frac{\left(5 e^{1.5 T}-10\right)^{2}}{0.64 T} \\
& \times \int_{0}^{T} \frac{u(t-0.5)}{u(t)\left[\left(e^{-1.5(t-0.5-(n+1) T)}+e^{1.5 T}-2\right)^{2}+\left(5 e^{1.5 T}-10\right)^{2}\right]} \mathrm{d} t,
\end{aligned}
$$

where $u(t) \in C_{T}$.
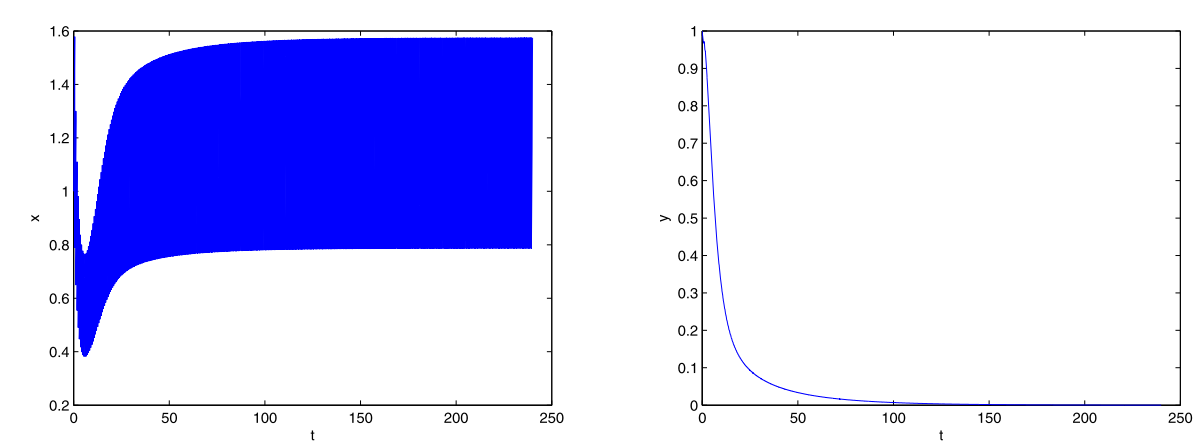

Figure 1 Time series of the solution of system (5.1) with $T=0.6$ 


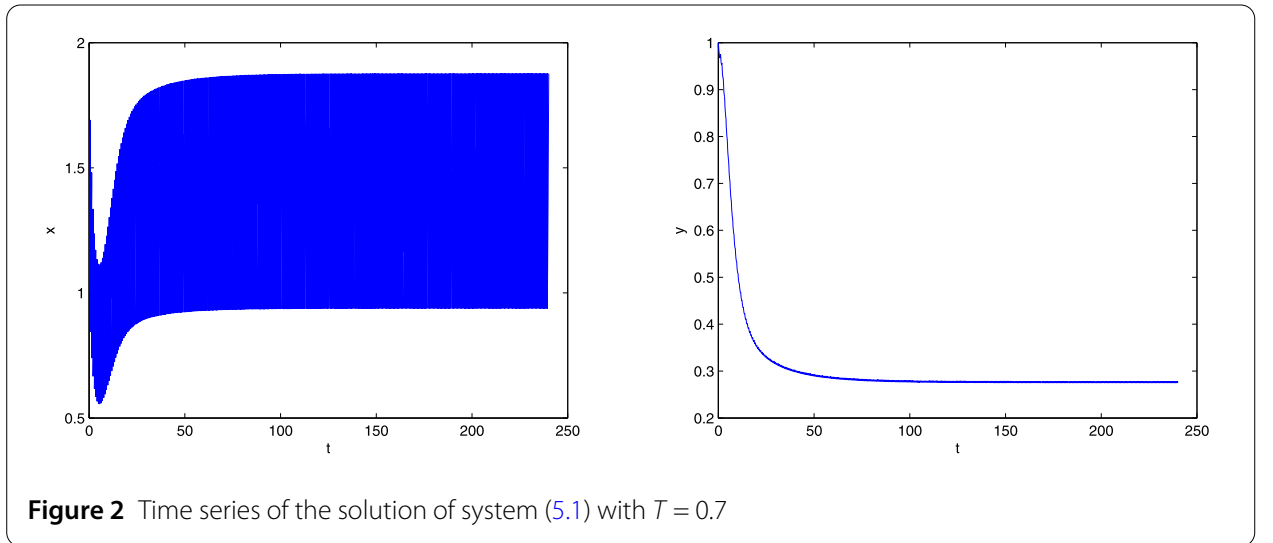

In system (5.1), let $T=0.6 \in\left(T_{1}^{*}, T_{2}^{*}\right)$. We compute the basic reproduction number $R_{0}$ numerically to get $R_{0} \approx 0.8590<1$. We also numerically compute the threshold $T^{*} \approx$ 0.6445 . It is obvious that $T=0.6<T^{*}$, that is, $R_{0}<1$. By Theorem 4.1 we obtain that the predator-free periodic solution is globally attractive. Figure 1 illustrates our theoretical results.

Let $T=0.7 \in\left(T_{1}^{*}, T_{2}^{*}\right)$. We numerically compute the basic reproduction number $R_{0} \approx$ $1.1250>1$. It is clear that $T=0.7>T^{*}$, that is, $R_{0}>1$. By Theorem 4.2 we obtain that the predator will become permanent. Figure 2 illustrates our theoretical results.

\section{Conclusion}

In this paper, we mainly discuss the extinction and permanence of the predator for system (1.1). Using the basic reproduction number coming from epidemiology, we may find the threshold value $R_{0}$ such that if $R_{0}<1$, then the predator is extinct, whereas if $R_{0}>1$, then it will persist. Thus we improve the results of [1]. As far as we know, this is the first paper employing this approach of [13] in ecology.

\section{Acknowledgements}

We would like to thank the anonymous referees very much for their valuable comments and suggestions.

\section{Funding}

The research was supported by the National Natural Foundation of China (11271371, 51479215, 11571324).

\section{Competing interests}

The authors declare that they have no competing interests.

\section{Authors' contributions}

Both authors contributed equally to the writing of this paper. Both authors read and approved the final manuscript.

\section{Author details}

'Department of Mathematics, North University of China, Taiyuan, P.R. China. ${ }^{2}$ School of Mathematics and Statistics, Central South University, Changsha, P.R. China.

\section{Publisher's Note}

Springer Nature remains neutral with regard to jurisdictional claims in published maps and institutional affiliations.

Received: 14 May 2018 Accepted: 19 November 2018 Published online: 07 December 2018

\section{References}

1. Li, S.Y., Liu, W.W.: A delayed Holling type III functional response predator-prey system with impulsive perturbation on the prey. Adv. Differ. Equ. 2016, 42 (2016). https://doi.org/10.1186/s13662-016-0768-8

2. Kuang, Y.: Delay Differential Equations with Applications in Population Dynamics. Academic Press, New York (1993)

3. May, R.M.: Stability and Complexity in Model Ecosystems. Princeton University Press, Princeton (2001) 
4. Murdoch, W.W., Briggs, C.J., Nisbet, R.M.: Consumer-Resource Dynamics. Princeton University Press, Princeton (2003)

5. Ruan, S.G.: On nonlinear dynamics of predator-prey models with discrete delay. Math. Model. Nat. Phenom. 4, 140-188 (2009)

6. Hui, J., Zhu, D.: Dynamic complexities for prey-dependent consumption integrated pest management models with impulsive effects. Chaos Solitons Fractals 29, 233-251 (2006)

7. Liu, B., Zhang, Y., Chen, L.S.: The dynamical behaviors of a Lotka-Volterra predator-prey model concerning integrated pest management. Nonlinear Anal., Real World Appl. 6, 227-243 (2005)

8. Zhang, H., Georgescu, P., Chen, L.S.: An impulsive predator-prey system with Beddington-Deangelis functional response and time delay. Int. J. Biomath. 1, 1-17 (2008)

9. Shao, Y.F., Li, Y:: Dynamical analysis of a stage structured predator-prey system with impulsive diffusion and generic functional response. Appl. Math. Comput. 220, 472-481 (2013)

10. Liu, X., Ballinger, G.: Boundedness for impulsive delay differential equations and applications to population growth models. Nonlinear Anal., Theory Methods Appl. 53, 1041-1062 (2003)

11. Leonid, B., Elena, B.: Linearized oscillation theory for a nonlinear delay impulsive equation. J. Comput. Appl. Math. 161, 477-495 (2003)

12. Yan, J.: Stability for impulsive delay differential equations. Nonlinear Anal., Theory Methods Appl. 63, 66-80 (2005)

13. Bai, Z.G.: Threshold dynamics of a time-delayed SEIRS model with pulse vaccination. Math. Biosci. 269, 178-185 (2015)

14. Garrione, M., Rebelo, C.: Persistence in seasonally varying predator-prey systems via the basic reproduction number. Nonlinear Anal., Real World Appl. 30, 73-98 (2016)

15. Pielou, E.C.: Introduction to Mathematical Ecology. Wiley-Interscience, New York (1969)

16. Paul, G., Hsieh, Y.H., Zhang, H.: A Lyapunov functional for a stage-structured predator-prey model with nonlinear predation rate. Nonlinear Anal., Real World Appl. 11, 3653-3665 (2010)

17. Bate, A.M., Hilker, F.M.: Predator-prey oscillations can shift when diseases become endemic. J. Theor. Biol. 316, 1-8 (2013)

18. Xu, R., Tian, X.H.: Global dynamics of a delayed eco-epidemiological model with Holling type-III functional response. Math. Methods Appl. Sci. 37, 2120-2134 (2014)

19. Bacaër, N., Guernaoui, S.: The epidemic threshold of vector-borne diseases with seasonality. J. Math. Biol. 53, 421-436 (2006)

20. Zhao, X.Q.: Basic reproduction ratios for periodic compartmental models with time delay. J. Dyn. Differ. Equ. (2015). https://doi.org/10.1007/s10884-015-9425-2

\section{Submit your manuscript to a SpringerOpen ${ }^{\circ}$ journal and benefit from:}

- Convenient online submission

- Rigorous peer review

- Open access: articles freely available online

- High visibility within the field

- Retaining the copyright to your article

Submit your next manuscript at $\gg$ springeropen.com 